\title{
Implementing TeamSTEPPS in a Shanghai COVID-19 Quarantine Center for Medical Observation to Enhance Interprofessional Attitude and Skills Regarding Teamwork
}

\author{
Tang Baoxin ${ }^{1, \dagger}$, Yu Haiping ${ }^{2}{ }^{\dagger}$, Gu Yanfen $^{3}$, Sun Xiaoliang ${ }^{4}$, Kang Baoli $^{4}$, Zhou Jian ${ }^{1}$, Wen Jiling ${ }^{5}$, \\ Yun Ying Hung ${ }^{6}$, Chen $\mathrm{Chi}^{7, *}$ \\ ${ }^{1}$ Department of Cardiovascular Medicine, Shanghai East Hospital, School of Medicine, Tongji University, Shanghai, China \\ ${ }^{2}$ Department of Nursing, Shanghai East Hospital, School of Medicine, Tongji University, Shanghai, China \\ ${ }^{3}$ Department of VIP Ward, Shanghai East Hospital, School of Medicine, Tongji University, Shanghai, China \\ ${ }^{4}$ Teaching and Training Department, Shanghai East Hospital, School of Medicine, Tongji University, Shanghai, China \\ ${ }^{5}$ Department of Urology, Shanghai East Hospital, School of Medicine, Tongji University, Shanghai, China \\ ${ }^{6}$ International \& Overseas Affairs, Chung Hwa University of Medical Technology, Taiwan, China \\ ${ }^{7}$ Teaching and Training Office, Shanghai East Hospital, School of Medicine, Tongii University, Shanghai, China
}

Email address:

99540047@qq.com (Tang Baoxin), pingping670@sina.com (Yu Haiping)), ariel611706@live.cn (Gu Yanfen), sunxl_sherry@126.com (Sun Xiaoliang),fkfinnkang@gmail.com (Kang Baoli),zj9758@126.com (Zhou Jian), wenjiling@tongji.edu.cn (Wen Jiling),kyyh7@hotmail.com (Yun Ying Hung), chichen2003@163.com (Chen Chi)

${ }^{*}$ Corresponding author

$\uparrow$ Tang Baoxin and Yu Haiping are co-first authors.

\section{To cite this article:}

Tang Baoxin, Yu Haiping, Gu Yanfen, Sun Xiaoliang, Kang Baoli, Zhou Jian, Wen Jiling, Yun Ying Hung, Chen Chi. Implementing TeamSTEPPS in a Shanghai COVID-19 Quarantine Center for Medical Observation to Enhance Interprofessional Attitude and Skills Regarding Teamwork. International Journal of Health Economics and Policy. Vol. 6, No. 3, 2021, pp. 85-91. doi: $10.11648 /$ j.hep.20210603.12

Received: July 13, 2021; Accepted: August 3, 2021; Published: August 11, 2021

\begin{abstract}
Objective: To improve the cooperation efficiency of an anti-epidemic team and achieve infection control and safety management in an observation site by implementing the TeamSTEPPS (Team Strategies \&Tools to Enhance Performance and Patient Safety Course) program. Methods: There were 41 staff members from hospitals, the district government, a health supervision institute, the Center for Disease Control and Prevention, public security, and hotels who participated in this program. The course was taught by a master trainer certified by The Agency for Healthcare Research and Quality (AHRQ). The project was evaluated by the Kirkpatrick Model which Including Level 1-reaction assessment (participants satisfaction), Level 2-learning assessment (team work attitude and cognition level), Level 3-behavior assessment (TeamSTEPPS tools and strategies using in workplace) and Level 4- results assessment (infection rate of isolation personnel and staff, incidence of safe adverse events, satisfaction of TeamSTEPPS practice in work). Results: After training and implementation of the TeamSTEPPS program, participants' attitudes, perception, knowledge, and skills regarding teamwork, team structure, leadership, situation monitoring, mutual support, and communication had significantly increased $(\mathrm{P}<0.01)$. Participants' satisfaction with the course was $99.02 \%$ and the application of TeamSTEPPS tools was effective. The actual application satisfaction of staff was $100 \%$. Until now, the infection rate and the incidence of safety adverse events in this observation site were $0 \%$. Conclusion: The TeamSTEPPS program is an effective strategy to improve interprofessional teamwork at the observation site, which contributes to safe COVID-19 management.
\end{abstract}

Keywords: TeamSTEPPS, Medical Observation Site, Infection Control, Safety Management 


\section{Introduction}

Since December of 2019, multiple cases of coronavirus (COVID-19) have been found in Wuhan, Hubei Province, China, and the epidemic has spread rapidly throughout the whole country. As of January 8, 2021, a total of 85,002,000 confirmed cases have been reported throughout the world [1]. Shanghai has established medical observation sites requisitioned by the government to provide overseas travelers 14 days quarantine. According to Chinese policies [2], all personnel coming to Shanghai from overseas must undergo isolation observation for 14 days, during which COVID-19 PCR detection will be conducted twice. People with symptoms will be sent to a designated hospital for further observation.

However, interprofessional team members at the observation site are temporarily assembled from various systems (including hospitals, the district government, health inspection centers, the CDC, public security, hotels, etc.) In the beginning, poor teamwork in the medical observation units was observed. This included an unclear distribution of labor, indistinct responsibilities, misunderstandings due to different professional backgrounds, difficulties reaching consensus, and interpersonal conflicts. These problems threatened staff and patient safety and made it more difficult to provide patient care.

The TeamSTEPPS program was adopted at the observation site to improve team performance, control infection risk, reduce errors, and ensure patient safety. TeamSTEPPS (Team Strategies \& Tools to Enhance Performance and Patient Safety Course) is a course developed by the Department of Defense (DoD) and The Agency for Healthcare Research and Quality (AHRQ), which is dedicated to improving teamwork among healthcare providers. Shanghai East Hospital introduced this course in 2015. The mentor team was composed of 19 AHRQ certified master trainers who have educated more than 5,000 medical staff in the last five years. Previous studies have shown that TeamSTEPPS training improves infection prevention and control, enhances team members' self-efficacy, reduces anxiety, improves monitoring awareness and teamwork skills (team structure, communication, leadership, mutual assistance, and monitoring), and significantly improves the ability to cope with infectious diseases [3-5]. Therefore, we utilized the TeamSTEPPS program at one observation site and achieved good results.

\section{Methods}

\subsection{Setting}

Our setting was a five-star hotel with 329 rooms located near the airport in Pudong, Shanghai. This hotel was one of the earliest to be commandeered by the government as an observation site in Shanghai. This site was chosen as the pilot based on the following considerations: the team members working at this site didn't know one another, and thus experienced communication problems and teamwork failures; there was one master TeamSTEPPS trainer at this site, and she was appointed team leader; and the governors in charge of anti-COVID19 management at this site were looking for efficient ways of leading multiple professionals as a team.

\subsection{Participants}

There were 41 staff members at the observation site who participated in this program. They hailed from seven different systems: hospitals, district government, a health supervision institute, the CDC, public security, hotels, and logistics support. All participants lived at the hotel. All of the participants voluntarily agreed to take part in this program.

\subsection{Methods}

The TeamSTEPPS 2.0 training program was conducted by a leader with master trainer certification from AHRQ, which lasted for eight hours (Figure 1). The contents of this training included: Team Structure, Communication tools (SBAR, Call-Out, Check-Back, Handoff, and I PASS THE BATON), Leadership (Brief, Huddle, and Debrief), Situation Monitoring (STEP, Cross-Monitoring, and the I'M SAFE checklist), and Mutual Support (Task Assistance, Feedback, Advocacy and Assertion, Two-Challenge Rule, CUS, and the DESC Script) [6]. The teaching materials included PPT, videos, and the Pocket Guide of Team Strategies \& Tools to Enhance Performance, which was authorized by AHRQ and translated by Shanghai East Hospital.

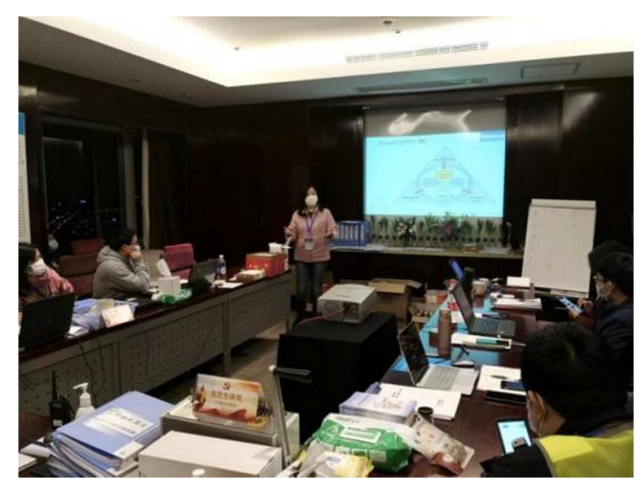

Figure 1. TeamSTEPPS program training.

\subsection{Project Implementation}

\subsubsection{Application}

The team members actively applied the TeamSTEPPS tools in their work. The master trainer monitored team performance, gave feedback and guidance, and continuously enabled the whole team to improve their teamwork by using five main skills: team structure, communication, leadership, mutual assistance, and monitoring [7]. For example, the division of labor was clearer after training. The medical staff was responsible for admitting and arranging isolators (Figure 2). The hotel staff was responsible for food delivery and garbage disposal (Figure 3), while the CDC staff was responsible for data collection and reporting. The public 
security staff was responsible for isolator safety, the health supervision staff was responsible for disinfection monitoring (Figure 4), while government staff was responsible for receiving information about the isolators. The isolators uploaded their body temperature through an app twice a day and promptly reported their signs and symptoms. Close contacts had their temperature taken and symptoms inquired about by medical staff (Figure 5).

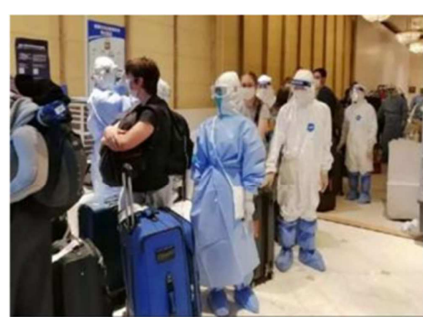

(a)

Figure 2. Admitting and arranging the isolators.

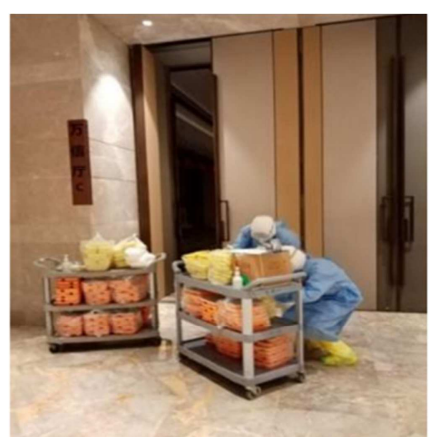

(a)

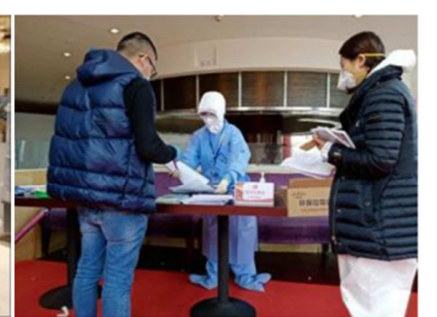

(b)

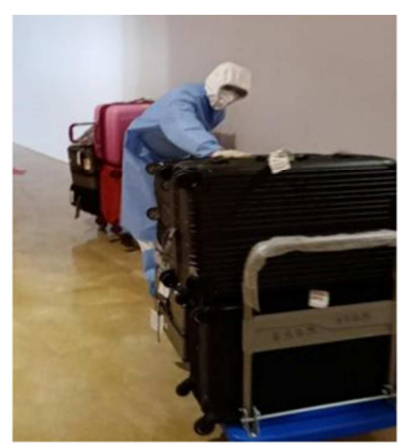

(b)
Figure 3. Food delivery and garbage disposa.

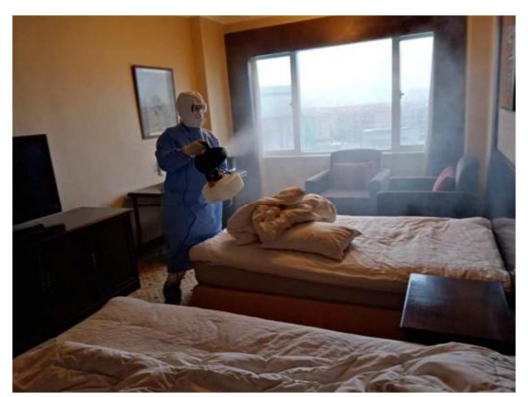

Figure 4. Disinfection monitoring.

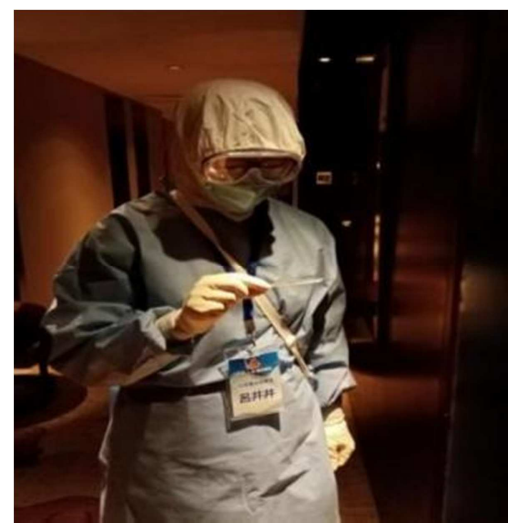

Figure 5. Monitoring temperatures and related symptoms.

\subsubsection{Evaluation}

We used the Kirkpatrick Model to evaluate program effectiveness [8]. The Kirkpatrick Model divides the training effect into four progressive levels based on the depth and difficulty of the evaluation. The first level is the reaction evaluation. The TeamSTEPPS course training satisfaction questionnaire was issued at the end of the training.

The second level is the learning evaluation. The Teamwork Perceptions Questionnaire (T-TPQ) and the Teamwork Attitudes Questionnaire (T-TAQ) were issued before and after training. T-TPQ includes five dimensions: structure, leadership, situational monitoring, mutual assistance, and communication, with a total of 35 items. The higher the score, the higher the team's cooperation abilities. T-TAQ is also a Likert 5-point scale with 30 items in five dimensions. The Cronbach's alpha coefficient for T-TPQ and T-TAQ were 0.949 and 0.938 , respectively, and the test-retest reliability was 0.959 and 0.964 , respectively. The construct validity was good [9].

The third level is the behavior evaluation. The team leader assessed team performance four weeks after training according to the TeamSTEPPS team performance evaluation form. The team leader also provided feedback and guidance.

The fourth level is the result evaluation. This included an evaluation of the infection rate of isolation personnel and staff, the incidence of adverse events, and results of the TeamSTEPPS tools application satisfaction questionnaire.

\subsection{Data Analysis}

Descriptive statistics were used for general information and to calculate a percentage, mean, standard deviation, etc. The t-test was used to compare data before and after the training. All tests were considered significant with a threshold of $0.05 \%$.

\section{Results}

\subsection{General Demographic Information}

From March 8 to April 3, 2020, the master trainer instructed 41 staff at the observation site (Table 1).

Table 1. General Demographic Information.

\begin{tabular}{llll}
\hline Item & Content & Number & Proportion (\%) \\
\hline \multirow{3}{*}{ Aender } & Male & 22 & 53.66 \\
& Female & 19 & 46.34 \\
& $20-30$ & 11 & 26.83 \\
& $31-40$ & 22 & 53.66 \\
& $41-50$ & 8 & 19.51 \\
& Doctor & 5 & 12.20 \\
& Nurse & 9 & 21.96 \\
& Government officer & 4 & 9.76 \\
& Health supervisor & 1 & 2.44 \\
& CDC & 5 & 12.20 \\
& Police officer & 5 & 9.76 \\
Education & Hotel & 4 & 12.20 \\
Background & logistics support & 8 & 19.48 \\
& High School & 7 & 17.07 \\
& College & 9 & 14.63 \\
& Undergraduate & 16 & 46.34 \\
& Postgraduate & 9 & 21.96 \\
\hline
\end{tabular}




\subsection{Kirkpatrick Model Level 1 - Reaction (TeamSTEPPS Training Satisfaction)}

All 41 participants provided feedback after the class. The results showed that the participants' satisfaction (including strongly agree and agree) with the course was $99.02 \%$
(Fiureg 6). Participants indicated that the TeamSTEPPS course was professional, practical, and improved their competencies. They reported that the teamwork tools were easy to understand, which facilitated communication and clarified their responsibilities.

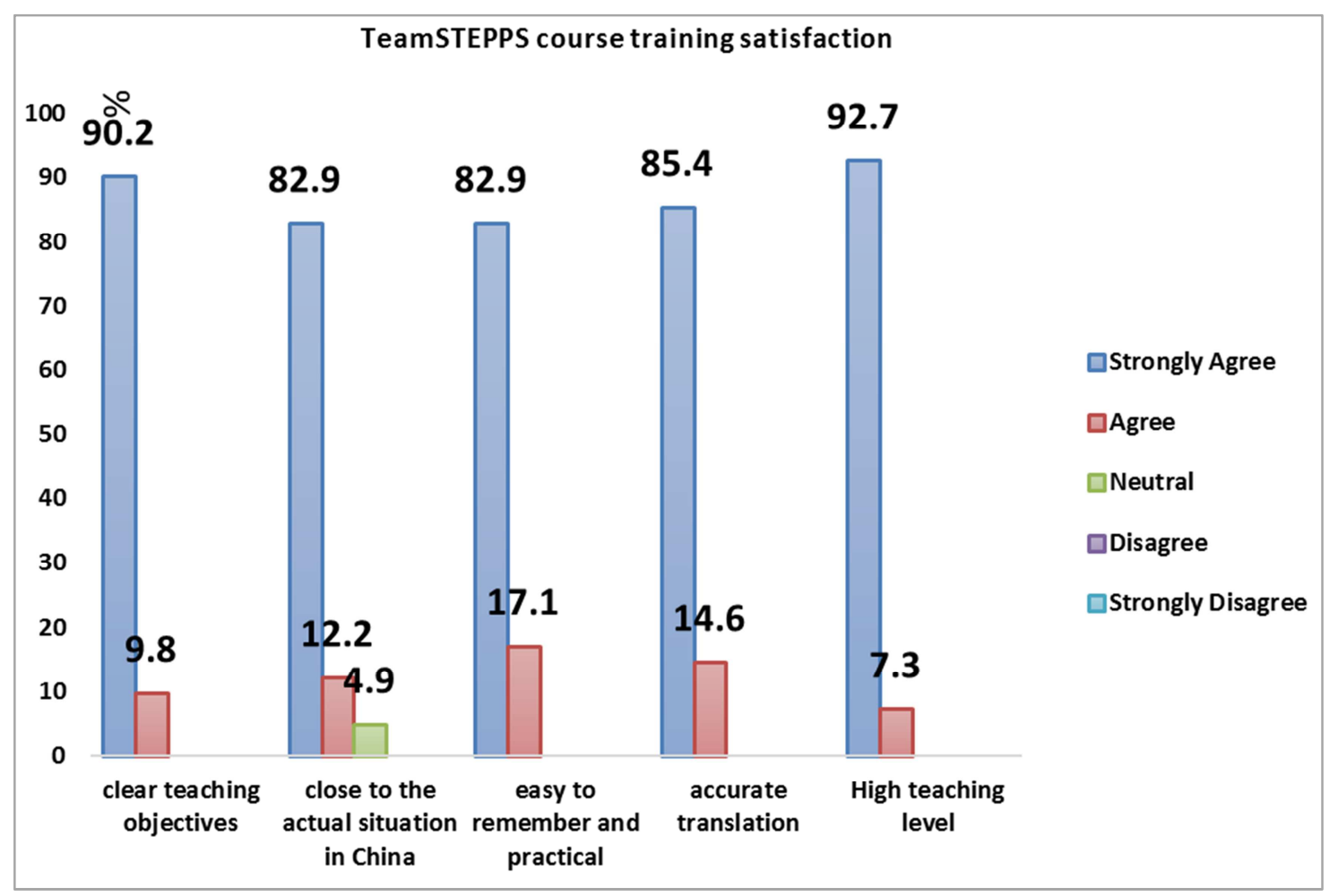

Figure 6. TeamSTEPPS training satisfaction (\%).

\subsection{Kirkpatrick Model Level 2 - Learning (Differences in Teamwork Perceptions and Attitudes Before and After Training)}

The Teamwork Perceptions Questionnaire (T-TPQ) and Teamwork Attitudes Questionnaire (T-TAQ) were used to assess learning (Table 2 and Table 3). After training, participants' attitudes and perceptions towards team structure, leadership, situation monitoring, mutual support, and communication had significantly increased $(\mathrm{P}<0.01)$.

Table 2. Team members' perceptions pre- and post-training $(x \pm s)$.

\begin{tabular}{|c|c|c|c|c|c|}
\hline & Team structure & Leadership & Situation monitoring & Mutual support & Communication \\
\hline Pre-training & $28.23 \pm 3.13$ & $29.65 \pm 4.51$ & $29.31 \pm 5.90$ & $28.38 \pm 5.49$ & $29.35 \pm 5.77$ \\
\hline Post-training & $32.88 \pm 6.80$ & $32.08 \pm 4.55$ & $31.19 \pm 4.96$ & $31.19 \pm 4.60$ & $30.92 \pm 4.44$ \\
\hline $\mathrm{t}$ & 3.00 & 7.20 & 6.29 & 5.63 & 6.37 \\
\hline
\end{tabular}

Table 3. Team members'attitudes pre- and post-training $(x \pm s)$.

\begin{tabular}{|c|c|c|c|c|c|}
\hline & Team structure & Leadership & situation monitoring & mutual support & communication \\
\hline Pre-training & $24.92 \pm 4.76$ & $25.04 \pm 5.79$ & $25.73 \pm 5.26$ & $20.31 \pm 2.95$ & $24.31 \pm 4.05$ \\
\hline Post-training & $28.00 \pm 2.86$ & $28.5 \pm 2.89$ & $28.19 \pm 2.88$ & $20.81 \pm 2.87$ & $26.08 \pm 2.58$ \\
\hline $\mathrm{t}$ & 2.73 & 2.64 & 3.55 & 4.42 & 3.95 \\
\hline $\mathrm{p}$ & 0.01 & 0.01 & 0.00 & 0.00 & 0.00 \\
\hline
\end{tabular}

\subsection{Kirkpatrick Model Level 3 - Behavior (Implementation of TeamSTEPPS Tools and Strategies at the Observation Site)}

\subsubsection{Clarifying the Team Structure}

All staff lived onsite and met briefly twice a day. The management structure included one team leader, two deputy team leaders, and one secretary. All of the team members were divided into 14 groups according to work requirements. Each group had one team leader (Figure 7). 


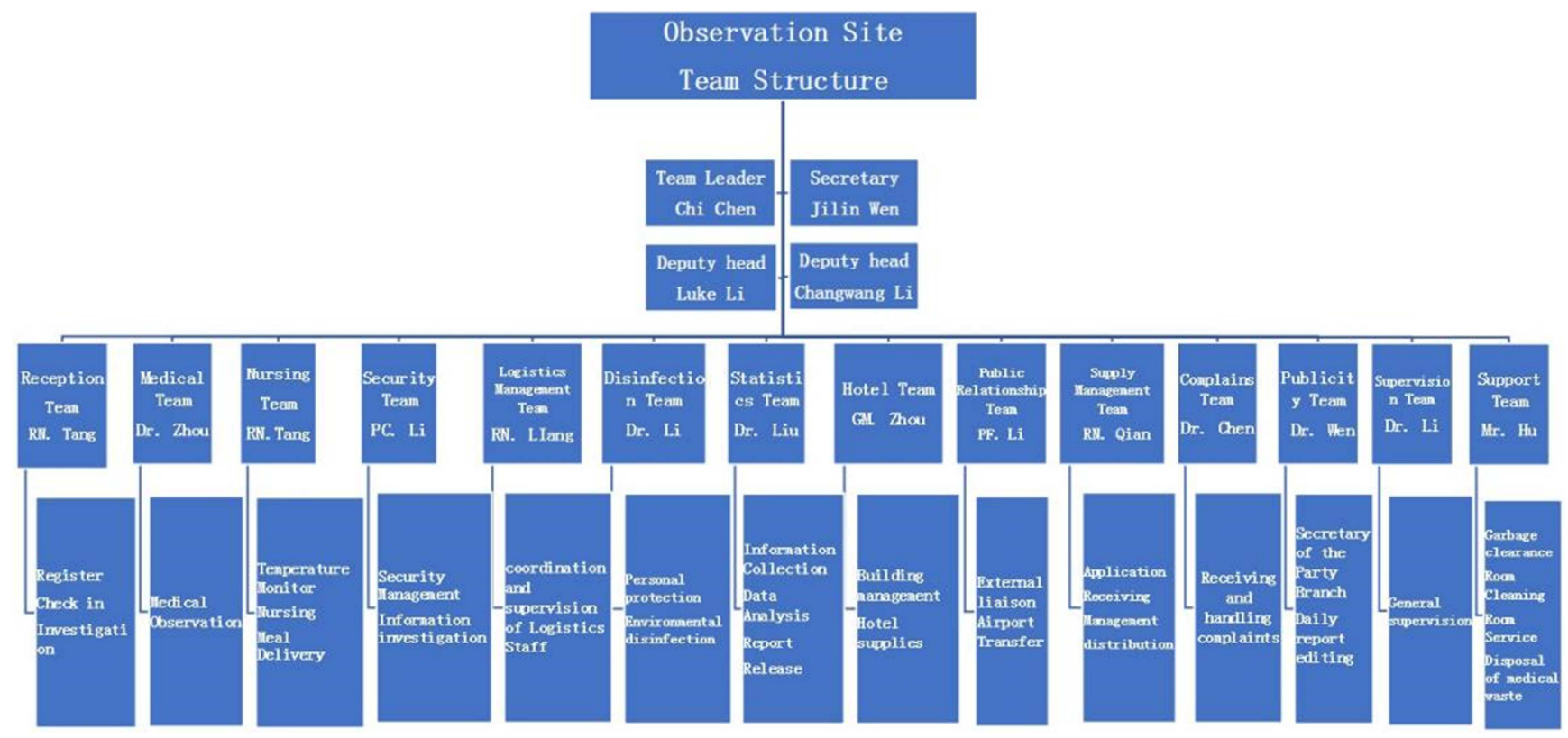

Figure 7. Organization structure of the observation site.

\subsection{2. "SBAR" as a Daily Communication Model}

Before the training, there were many communication problems, including incomplete or lost information and complaints without constructive comments, which lead to confusion and arguments. Therefore, the team leader required that all team members use the SBAR (Situation-Background-Assessment-Recommendation) tool in their daily communication. SBAR reduced incomplete, unclear, and imprecise communication [10]. This tool allowed members to quickly engage in structural thinking and expression.

\subsection{3. “Brief, Huddle, and Debrief” for Mental Sharing}

The concept of a "Brief" in the morning and a "Debrief" in the evening allowed for plan sharing, problem-solving, and the organization of daily work. During these meetings, team members could discuss any problems. "Huddle" was used for emergent events. These three tools prevented errors, ensured safety, and improved work quality.

\subsection{4. "STEP" to Monitor High-risk Personnel}

"STEP", a tool for situation monitoring, includes four components: the status of patients, the status of team members, the environment, and progress towards a goal [11]. We used STEP to monitor high-risk isolators, including the elderly, young children and infants, and those with chronic diseases (hypertension and diabetes), mental illness (claustrophobia syndrome, anxiety, and depression). Medical staff called these patients every day to confirm their health condition. An ambulance was called immediately if necessary. Adverse safety events did not occur onsite in these high-risk groups.

\subsection{5. "I'M SAFE Checklist" for Self-monitoring}

Staff had to monitor and report their temperature twice a day. Members used the "I'M SAFE checklist", which included monitoring temperature, symptoms, and mental status. One member of the medical staff collected this information and reported on it at the daily debrief meeting. The team leader was aware of any reported symptoms, and measures could be carried as soon as possible.

\subsection{6. “CUS” for Interpersonal Conflicts [12]}

We used "CUS" to express concerns about several unsafe incidents. CUS indicates "I am Concerned, I feel Uncomfortable, and this is a Safety event." On March 31, a staff member from the CDC indicated that isolation time calculation was not accurate, as it should be accurate to hours, not days. He used the CUS to express his opinion. All of the team members discussed this issue in the debrief meeting. The staff member emphasized that this issue was related to infection prevention and safety management, and insisted that the current procedure be adjusted. After his persistence and explanations, all of the team members agreed to change the process. To ensure safety, everyone should be encouraged to use the "CUS" tool to "Stop The Line" when they discover safety concerns.

\subsection{Kirkpatrick Model Level 4 - Results Evaluation}

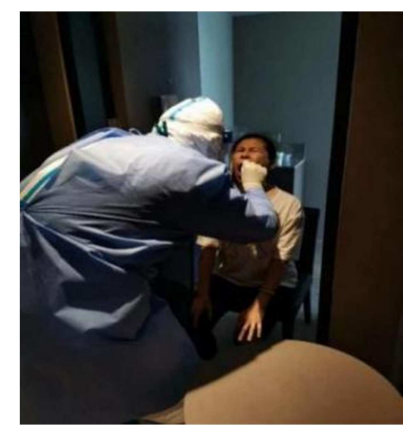

Figure 8. COVID-19 PCR tests.

From March 8 to April 3, 2020, team members admitted 702 isolators, including 33 individuals from foreign countries and 11 close contacts. COVID-19 PCR tests were carried out before the 
staff and the isolators left the site (Figure 8). The infection rate onsite was $0 \%$. The incidence of adverse safety events was $0 \%$, and the satisfaction score regarding the practical application of the TeamSTEPPS tool was $100 \%$ (Figure 9).

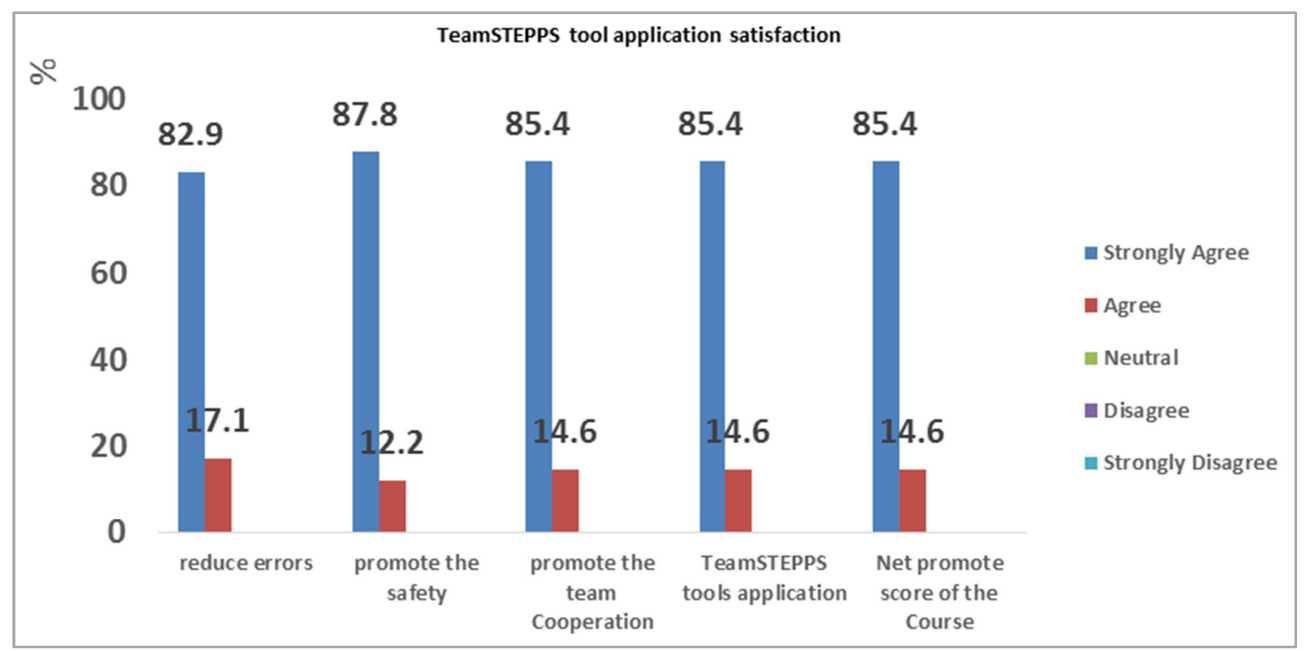

Figure 9. Satisfaction regarding the practical application of the TeamSTEPPS tools (\%).

\section{Discussion}

\subsection{TeamSTEPPS Improves Cooperation and Teamwork}

The World Health Organization (WHO) declared the outbreak of the novel coronavirus (2019-nCoV) a public health emergency of international concern [13], which poses a great challenge to each nation's healthcare system. An efficient interprofessional team is required for infection prevention and control and adequate safety management at COVID observation sites. Our research confirmed that the TeamSTEPPS 2.0 course improved the performance of a temporary assembly team. TeamSTEPPS tools and strategies were used to improve cooperation and promote the members' perceptions and attitudes regarding teamwork $(\mathrm{P}<0.01)$. No staff infections or adverse safety events occurred.

\subsection{Implementing the TeamSTEPPS Program}

The key points of successfully implementing the TeamSTEPPS training program in an observation site are:

1) emphasize infection control and safety management through training [14], and require members to follow this goal at all times;

2) integrate all relevant members into the team and encourage self-driving initiatives;

3) the master trainer must oversee training and support team members in using the TeamSTEPPS tools and strategies; and

4) a clear division of labor, quick feedback, and timely adjustments effectively promote team cooperation.

We found that the TeamSTEPPS tools helped create a workplace safety culture.

\subsection{Limitations and Future Training}

Satisfaction with the TeamSTEPPS training reached $99.02 \%$. Some members were not satisfied with the teaching equipment, and limited teaching conditions meant that the teaching methods were often based on oral techniques. Games, scenario simulations, and other methods could not be carried out. However, relevant research shows that simulation teaching improves team cooperation training [15]. Also, members without a medical background lacked personal protection and infection control knowledge, including the concepts of "clean area", "semi-polluted area," and "contaminated area." Although we arranged for a training on personal protective equipment (PPE) for all members (Figure 10), but cross-contamination still occurred. In the future, videos on this topic should be used in the course. In addition, cases of personal protection failure combined with teamwork tools like cross-monitoring and mutual support may generate interest in future training.

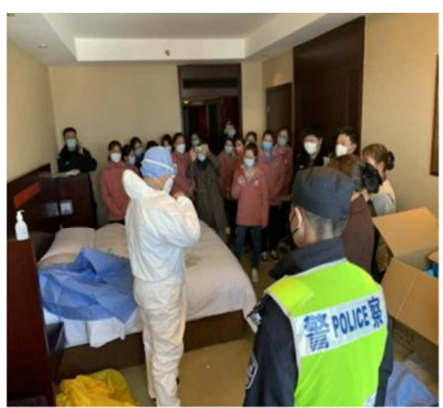

(a)

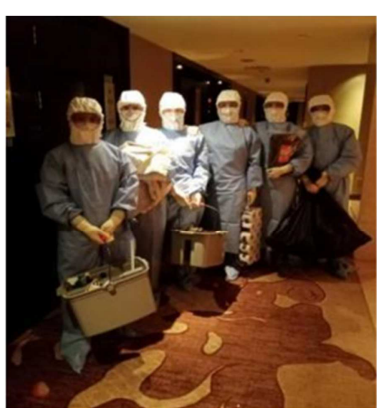

(b)
Figure 10. Personal protective equipment (PPE) training.

\section{Conclusion}

This is the first time that the TeamSTEPPS program has been used outside of a hospital or medical school. As the global epidemic spreads, observation sites become an important part of COVID-19 infection control and safety management. After implementing the TeamSTEPPS concept, teamwork, cooperation, and the safety culture significantly improved at the observation sites. Participants' attitudes, 
perception, knowledge, and skills regarding teamwork, team structure, leadership, situation monitoring, mutual support, and communication had significantly increased $(\mathrm{P}<0.01)$. Participants' satisfaction with the course was $99.02 \%$ and the application of TeamSTEPPS tools was effective. The actual application satisfaction of staff was $100 \%$. Until now, the infection rate and the incidence of safety adverse events in this observation site were $0 \%$. This suggests that TeamSTEPPS could enhance patient safety, especially in high-risk environments such as isolation observation sites.

\section{Acknowledgements}

The authors wish to thank the anonymous reviewers, associate editors, and editors for their thoughtful reviews and suggestions.

\section{References}

[1] COVID-19 Data Repository by the Center for Systems Science and Engineering (CSSE) at Johns Hopkins University. https://coronavirus.jhu.edu/map.html. Date accessed: November 21, 2020.

[2] COVID-19 risk level adjustment. http://www.gov.cn/xinwen/2020-05/02/content 5508271.htm. Date accessed: May 2, 2020.

[3] Marrs R, Horsley T L, Hackbarth D, et al. High consequence infectious diseases training using interprofessional simulation and TeamSTEPPS [J]. Am J Infect Control, 2019, 48 (6): 615-620.

[4] He J C, Golob J F, Brajcich B C, et al. Benefit of TeamSTEPPS Rounding improvement project on infection-related monitoring [J]. Surg Infect (Larchmt), 2016, 17 (5): 530-534.

[5] Aldawood F, Kazzaz Y, AlShehri A, et al. Enhancing teamwork communication and patient safety responsiveness in a paediatric intensive care unit using the daily safety huddle tool [J]. BMJ Open Qual, 2020, 9 (1).
[6] Ross JG, Meakim C, Hohenleitner SG. Outcomes of TeamSTEPPS Training in Prelicensure Health Care Practitioner Programs: An Integrative Review [J]. J Nurs Educ. 2020; 59 (11): 610-616. doi: 10.3928/01484834-20201020-03.

[7] Clancy CM, Tornberg DN. TeamSTEPPS: Assuring Optimal Teamwork in Clinical Settings [J]. Am J Med Qual. 2019; 34 (5): 436-438. doi: 10.1177/1062860619873181.

[8] De Gagne JC, Park HK, Hall K, Woodward A, Yamane S, Kim SS. Microlearning in Health Professions Education: Scoping Review [J]. JMIR Med Educ. 2019; 5 (2): e13997. Published 2019 Jul 23. doi: 10.2196/13997.

[9] Jie H, Haiping Y, Meiying Z, et al. Reliability and validity of the Chinese version of medical team cooperation cognition and attitude scale [J]. Chin J Mod Nurs, 2020, 26 (21): 2817-2823.

[10] Ruhomauly Z, Betts K, Jayne-Coupe K, et al. Improving the quality of handover: implementing SBAR. Future Healthc J. 2019; 6 (Suppl 2): 54. doi: 10.7861/futurehosp.6-2s-s54.

[11] Staines A, Lécureux E, Rubin P, Baralon C, Farin A. Impact of TeamSTEPPS on patient safety culture in a Swiss maternity ward [J]. Int J Qual Health Care. 2020; 32 (9): 618-624. doi: 10.1093/intqhe/mzz062.

[12] Piryani RM, Piryani S. Conflict Management in Healthcare [J]. J Nepal Health Res Counc. 2018; 16 (41): 481-482. doi: https://doi.org/10.33314/jnhrc. 1703 .

[13] WHO. Statement on the second meeting of the International Health Regulations (2005). Emergency committee regarding the outbreak of novel coronavirus (2019-nCoV). 30 January 2020. Available https://www.who.int/news-room/detail/30-01-2020-statementon-the-second-meeting-of-the-international-health-regulations -(2005)-emergency-committee regarding-the-outbreak-of-novel-coronavirus-(2019-nCoV). Date accessed: 6 February 2020.

[14] Jule JG. Workplace Safety: A Strategy for Enterprise Risk Management [J]. Workplace Health Saf. 2020; 68 (8): 360-365. doi: $10.1177 / 2165079920916654$.

[15] Clapper T C, Ching K, Lee J G, et al. A TeamSTEPPS (R) implementation plan for recently assigned interns and nurses. J Interprof Care, 2019, 33 (6): 823-827. 